\title{
Correlation of magnetic resonance diffusion tensor imaging parameters with American Spinal Injury Association score for prognostication and long-term outcomes
}

\author{
Saman Shabani, MD, Mayank Kaushal, MD, MBA, Matthew Budde, PhD, and \\ Shekar N. Kurpad, MD, PhD \\ Department of Neurosurgery, Medical College of Wisconsin, Milwaukee, Wisconsin
}

OBJECTIVE Conventional MRI is routinely used to demonstrate the anatomical site of spinal cord injury (SCI). Howev$\mathrm{er}$, quantitative and qualitative imaging parameters have limited use in predicting neurological outcomes. Currently, there are no reliable neuroimaging biomarkers to predict short- and long-term outcome after SCl.

METHODS A prospective cohort of 23 patients with $\mathrm{SCl}$ (19 with cervical SCI [CSCl] and 4 with thoracic SCI [TSCI]) treated between 2007 and 2014 was included in the study. The American Spinal Injury Association (ASIA) score was determined at the time of arrival and at 1 -year follow-up. Only 15 patients (12 with $\mathrm{CSCl}$ and 3 with TSCl) had 1-year follow-up. Whole-cord fractional anisotropy (FA) was determined at C1-2, following which C1-2 was divided into upper, middle, and lower segments and the corresponding FA value at each of these segments was calculated. Correlation analysis was performed between FA and ASIA score at time of arrival and 1-year follow-up.

RESULTS Correlation analysis showed a positive but nonsignificant correlation $(p=0.095)$ between FA and ASIA score for all patients ( $\mathrm{CSCl}$ and TCSI) at the time of arrival. Additional regression analysis consisting of only patients with $\mathrm{CSCl}$ showed a significant correlation $(p=0.008$ ) between FA and ASIA score at time of arrival as well as at 1-year follow-up $(p=0.025)$. Furthermore, in case of patients with CSCl, a significant correlation between FA value at each of the segments (upper, middle, and lower) of C1-2 and ASIA score at time of arrival was found ( $p=0.017, p=0.015$, and $p=$ 0.002 , respectively).

CONCLUSIONS In patients with CSCI, the measurement of diffusion anisotropy of the high cervical cord (C1-2) correlates significantly with injury severity and long-term follow-up. However, this correlation is not seen in patients with TSCl. Therefore, FA can be used as an imaging biomarker for evaluating neural injury and monitoring recovery in patients with CSCl.

https://thejns.org/doi/abs/10.3171/2018.12.FOCUS18595

KEYWORDS diffusion tensor imaging; spinal cord injury; fractional anisotropy; anisotropic diffusion

$\mathrm{S}$ PINAL cord injury (SCI) is a devastating condition with high morbidity and mortality and is characterized by partial or complete loss of neurological function below the site of injury due to disruption of spinal cord integrity. Conventional MRI is most commonly used for the radiological evaluation of the injured cord. The findings seen on anatomical MRI such as hemorrhage, lesion length, cord compression, and edema have been shown to have limited prognostic utility in predicting outcomes. . $^{3,13,21,22,25}$

Diffusion tensor imaging (DTI), a noninvasive imaging modality, uses the restricted diffusion of water molecules in neuronal tissue as the major contrast mechanism to provide quantitative information about microstructural integrity even within regions of the cord that appear nor-

ABBREVIATIONS ASIA = American Spinal Injury Association; $\mathrm{CSCI}=$ cervical $\mathrm{SCI} ; \mathrm{DTI}=$ diffusion tensor imaging; $\mathrm{DWI}=$ diffusion-weighted imaging; $\mathrm{FA}$ = fractional anisotropy; FOV = field of view; $\mathrm{ROI}$ = region of interest; $\mathrm{SCl}=$ spinal cord injury; $\mathrm{TSCl}=$ thoracic $\mathrm{SCl}$.

SUBMITTED October 31, 2018. ACCEPTED December 13, 2018.

INCLUDE WHEN CITING DOI: 10.3171/2018.12.FOCUS18595. 
mal on structural MRI. ${ }^{2,20,23,30}$ The restricted linear movement along the length of the spinal cord is due to barriers to diffusion such as axonal membranes and myelin sheath, which if interrupted manifest as alterations to the DTI parameters derived from the diffusion signal.

Studies in both acute and chronic SCI have demonstrated structural alterations remote from the site of injury in the form of changes to DTI indices. ${ }^{6,11,15,27,34,35}$ One such DTI parameter, fractional anisotropy (FA), measuring the degree of diffusion anisotropy or restricted diffusion, is often correlated with injury severity and/or neurological outcomes. Previously, our group reported an association between FA value estimated at the high cervical cord (C1-2) during the acute phase of SCI and American Spinal Injury Association (ASIA) grades at admission, which showed the utility of DTI in early detection of structural changes in white matter tracts. ${ }^{34}$ In the present analysis, we evaluate the relationship between FA determined from the high cervical cord (C1-2) and long-term functional outcome in patients with acute SCI.

\section{Methods}

\section{Patient Population}

Our study included 23 patients with acute SCI who underwent presurgical DTI and T2-weighted MRI of the cervical spine at our institution between March 2007 and December 2014. Of the 23 patients, 19 suffered from acute cervical SCI (CSCI) and 4 had acute thoracic SCI (TSCI). We performed a chart review to acquire relevant demographic details, neurological level of injury, and ASIA grades at admission. Prior to scanning, all patients provided written informed consent, and all the procedures were approved by the local institutional review board.

\section{Diffusion Tensor Imaging}

After giving their consent, the patients underwent DTI of the cervical spine (C1-T1) on a 1.5-T MR scanner (Signa Excite; GE Medical Systems) with a CTL spine coil. Sagittal T2-weighted images of the cervical spine were acquired in all subjects by using a TR/TE of 4000/102 msec, matrix size of $384 \times 224$, and field of view (FOV) of $20 \mathrm{~cm}^{2}$. The initial 6 subjects were recruited prior to May 2011 and were scanned on an older 1.5-T GE MR scanner using the following scanning parameters: 25 equidistant directions at a b-value of $500 \mathrm{sec} / \mathrm{mm}^{2}$, TR/TE of $4500 / 80$ msec, matrix size of $128 \times 128$, and FOV of $260 \mathrm{~mm}^{2}$. The remaining 17 subjects were imaged using the following scanning protocol: 15 distinct directions, b-value of $600 \mathrm{sec} / \mathrm{mm}^{2}$, TR/TE of 5000/98.2 msec, matrix size of $128 \times 128$, and FOV of $19 \mathrm{~cm}^{2}$. The data from both the old and new MR scanner were included in the present analysis. This decision was based on the comparison of mean FA and signal-to-noise ratios of an axial image at the C4-5 level for 4 healthy volunteers imaged on both scanners, which showed no significant difference in either parameter. ${ }^{34}$

\section{Image Processing}

Diffusion images were analyzed using the MedINRIA software package (www-sop.inria.fr/asclepios/software/
TABLE 1. Demographics of patients with acute $\mathrm{SCl}$

\begin{tabular}{|c|c|c|c|c|c|}
\hline $\begin{array}{l}\text { Case } \\
\text { No. }\end{array}$ & $\begin{array}{l}\text { Age } \\
\text { (yrs) }\end{array}$ & Sex & $\begin{array}{l}\text { Neurological } \\
\text { Level of Injury }\end{array}$ & $\begin{array}{l}\text { ASIA } \\
\text { Grade }\end{array}$ & $\begin{array}{c}\text { 1-Yr FU } \\
\text { ASIA Grade }\end{array}$ \\
\hline 1 & 53 & $M$ & C4 & $D$ & NA \\
\hline 2 & 73 & $M$ & C5 & $D$ & $\mathrm{D}$ \\
\hline 3 & 62 & $\mathrm{~F}$ & C5 & C & $C$ \\
\hline 4 & 20 & $M$ & $\mathrm{~T} 7$ & $A$ & $A$ \\
\hline 5 & 65 & $M$ & $\mathrm{C} 7$ & $D$ & $D$ \\
\hline 6 & 54 & $M$ & C4 & $A$ & NA \\
\hline 7 & 60 & $M$ & C3 & C & NA \\
\hline 8 & 54 & $M$ & $\mathrm{C} 7$ & $D$ & $\mathrm{D}$ \\
\hline 9 & 32 & $M$ & C5/6 & $D$ & NA \\
\hline 10 & 43 & $\mathrm{~F}$ & C6 & $D$ & $D$ \\
\hline 11 & 23 & $M$ & $\mathrm{C} 4$ & $D$ & D \\
\hline 12 & 56 & $M$ & T10 & $A$ & $D$ \\
\hline 13 & 52 & $M$ & $\mathrm{C} 2$ & $C$ & $C$ \\
\hline 14 & 45 & $\mathrm{~F}$ & C5 & B & $C$ \\
\hline 15 & 18 & $\mathrm{~F}$ & C5 & $D$ & C \\
\hline 16 & 63 & $\mathrm{~F}$ & $\mathrm{C} 5$ & $D$ & NA \\
\hline 17 & 24 & $M$ & $\mathrm{~T} 5 / 6$ & $A$ & $A$ \\
\hline 18 & 67 & $M$ & $\mathrm{C} 3 / 4$ & $C$ & NA \\
\hline 19 & 37 & $M$ & T5 & A & $A$ \\
\hline 20 & 20 & $M$ & C6 & $B$ & $B$ \\
\hline 21 & 64 & $\mathrm{~F}$ & $\mathrm{C} 7$ & B & B \\
\hline 22 & 69 & $\mathrm{~F}$ & $\mathrm{C} 3 / 4$ & C & NA \\
\hline 23 & 22 & $M$ & C6 & $D$ & NA \\
\hline
\end{tabular}

$\mathrm{FU}=$ follow-up; $\mathrm{NA}=$ not available.

MedINRIA). The estimation of FA was done on axial FA maps through $\mathrm{C} 1-2$ (high cervical cord), with each voxel that was inside the manually drawn regions of interest (ROIs) included in the calculation. Whole-cord ROIs were drawn within the perimeter of the cord so as to avoid partial volume effects due to CSF.

\section{Statistical Analysis}

Correlations between FA and ASIA grades at admission as well as at 1-year follow-up were analyzed using the Pearson correlation. Statistical analysis was performed using SPSS software version 20.0 (IBM Corp.). ASIA grades were converted to numerical values to conduct the correlational analysis, with ASIA A corresponding to the numerical value of 1 . Means were reported as \pm standard error of the mean, and the level of significance was set at $p<0.05$.

\section{Results}

The demographic data for each subject with SCI are shown in Table 1.

For the determination of a relationship between FA derived from the high cervical cord and ASIA grade at admission, separate analyses were conducted, with one comprising all subjects (both cervical and thoracic, $\mathrm{N}=$ 23) and the other containing only the cervical subjects (n = 19; Fig. 1). When the entire patient cohort was included, 


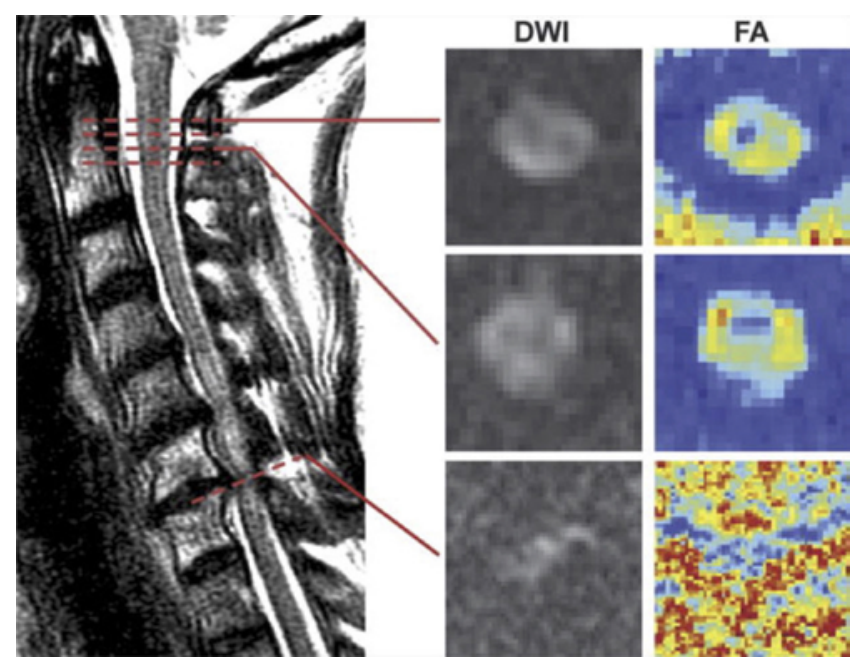

FIG. 1. Findings seen with conventional MRI as well as with DTI (FA maps) at the C1-2 level as well as the site of injury in a patient with $\mathrm{CSCl}$. The sagittal cervical spine T2-weighted image of a patient with $\mathrm{SCl}$ shows axial DWI and FA maps in the high cervical cord (rostral) and injury site (C6-7 level, caudal). The DW images obtained at the high cervical cord have better-defined cord delineation compared to the images obtained at the injury site. Reproduced with the permission of Oxford University Press from Vedantam et al: High cervical fractional anisotropy as an imaging marker for spinal cord injury. Neurosurgery 61 (Suppl 1):167-170, 2014.

FA of the whole cord showed a nonsignificant correlation with the ASIA grade on admission $(\mathrm{N}=23, \mathrm{p}=0.095$; Fig. 2). For only patients with CSCI, FA derived from the high cervical cord showed a significant correlation with ASIA grades $(n=19, p=0.008$; Fig. 3). Similarly, FA showed a nonsignificant correlation with ASIA grade at 1-year follow-up for the entire patient cohort $(\mathrm{N}=15, \mathrm{p}=0.589$; Fig. 4), whereas FA showed a significant correlation with ASIA

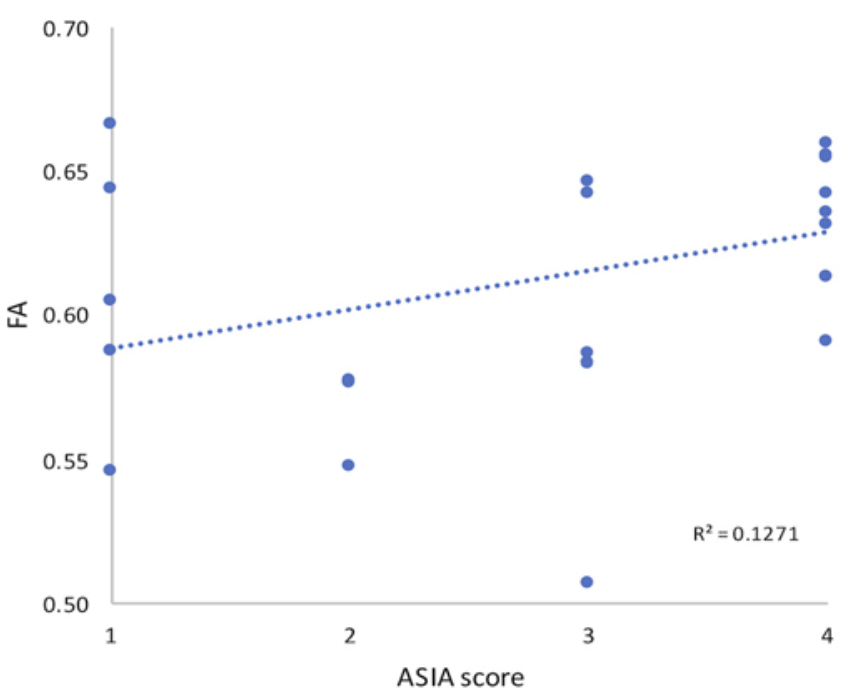

FIG. 2. Graph showing nonsignificant correlation between FA measured at the high cervical cord $(\mathrm{C} 1-2)$ and $\mathrm{ASIA}$ score at the time of admission $(p=0.095)$ for the group of patients with both $\mathrm{CSCl}$ and $\mathrm{TSCl}(\mathrm{N}=23)$. The ASIA grades were converted into numerical values, with ASIA A corresponding to the numerical value of 1 .

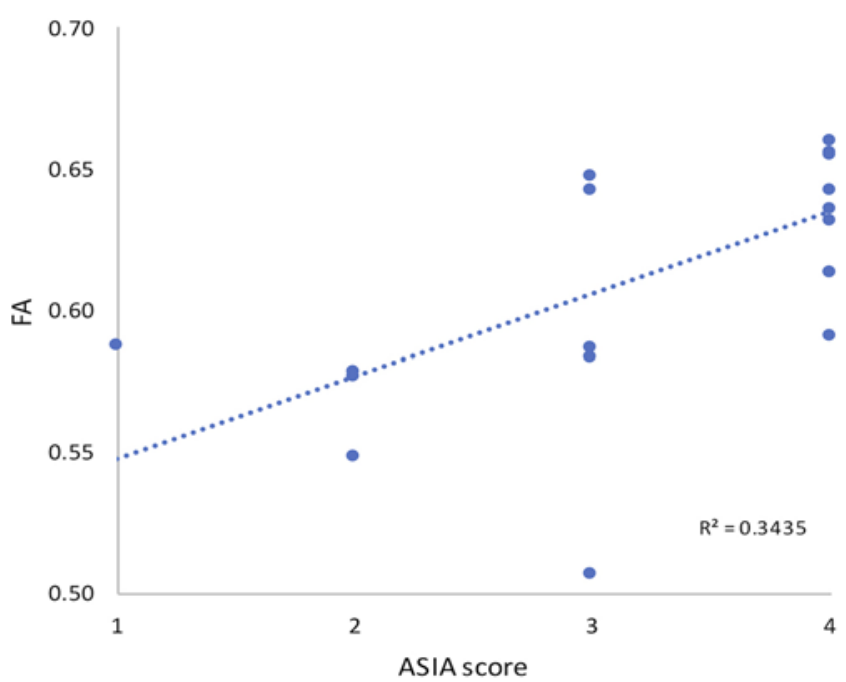

FIG. 3. Graph showing significant correlation between FA measured at the high cervical cord (C1-2) and ASIA score at the time of admission $(p=0.008)$ for the group of patients with only CSCI $(N=19)$. The ASIA grades were converted into numerical values, with ASIA A corresponding to the numerical value of 1 .

grade at 1-year follow-up when only patients with CSCI were included $(n=12, p=0.025$; Fig. 5$)$.

The high cervical cord (C1-2) was next divided into 3 segments (upper, middle, and lower) and FA of the whole cord for each segment was correlated with ASIA at admission for subjects with CSCI. FA obtained from each segment showed significant correlation with ASIA grades at admission, and the magnitude of the correlation increased along the rostrocaudal direction $(\mathrm{p}=0.017, \mathrm{p}=0.015$, and $\mathrm{p}=0.002$ for upper, middle, and lower segments of high cervical cord [C1-2], respectively; Fig. 6).

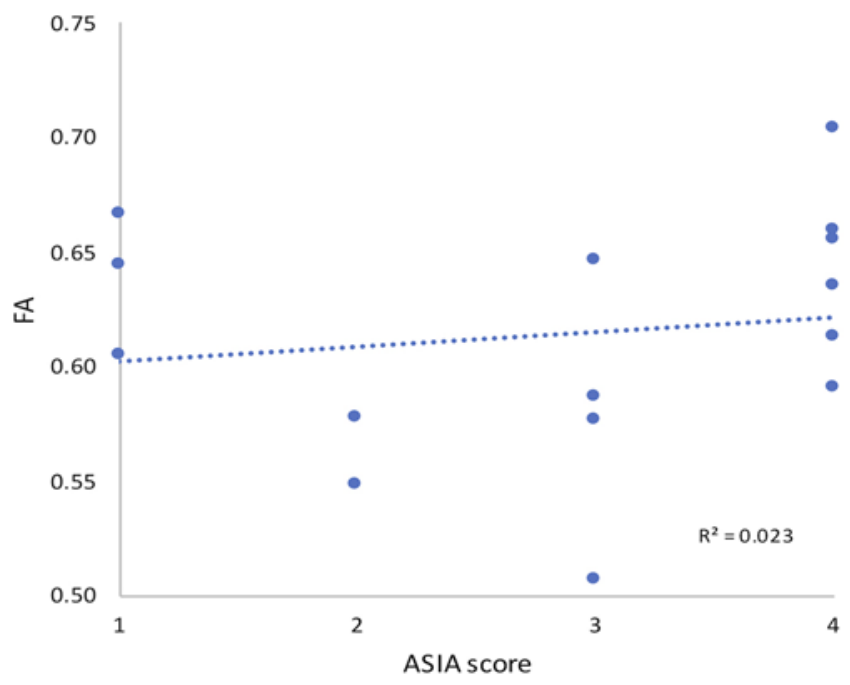

FIG. 4. Graph showing nonsignificant correlation between FA measured at the high cervical cord (C1-2) and ASIA score at 1-year follow-up for the group of patients with both $\mathrm{CSCl}$ and $\mathrm{TSCl}(\mathrm{p}=0.589)$. There was a total of $65 \%$ follow-up at 1 year $(\mathrm{N}=15)$. The ASIA grades were converted into numerical values, with ASIA A corresponding to the numerical value of 1 . 


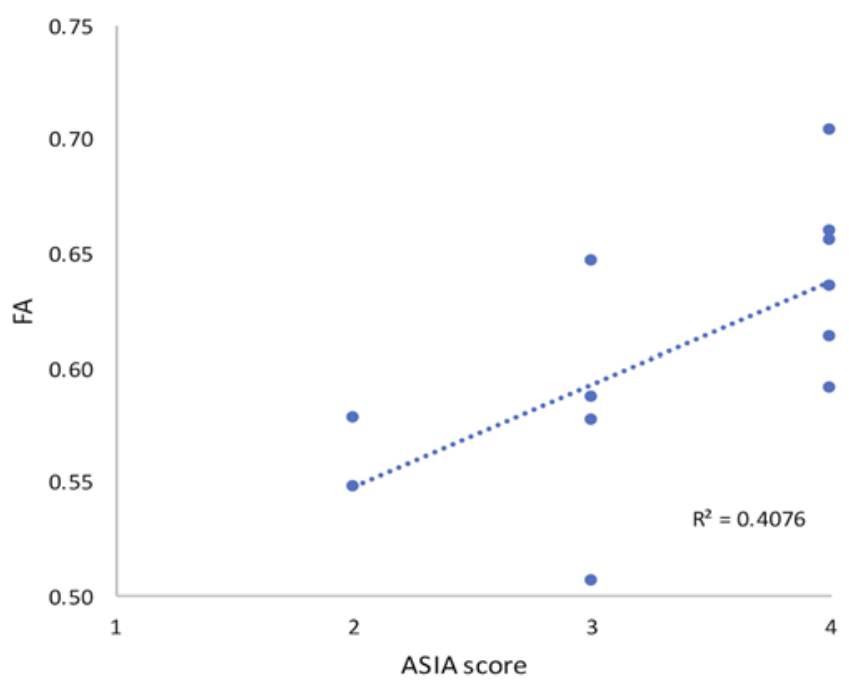

FIG. 5. Graph showing significant correlation between FA measured at the high cervical cord ( $\mathrm{C} 1-2)$ and ASIA score at 1-year follow-up for the group of patients with only CSCI $(p=0.025)$. There was a total of $63 \%$ follow-up at 1 year $(N=12)$. The ASIA grades were converted into numerical values, with ASIA A corresponding to the numerical value of 1 .

\section{Discussion}

The logistical challenges associated with imaging the spinal cord during the acute phase of SCI have limited the literature on DTI in human subjects and as a result, our understanding of the utility of DTI is informed by animal studies and the few human studies published so far., ${ }^{4,14,18-20 \text {, }}$ $23,24,28,30,31,33-36$ In the current scenario, the present study, comprising 23 patients, represents the largest collection of patients in whom DTI was done during the acute phase of SCI. We demonstrated a significant relationship between ASIA, which provides an estimate of white matter integrity and motor function in patients with SCI, and FA obtained from the upper cervical cord $(\mathrm{Cl}-2)$ in patients with CSCI. In addition, we found a significant correlation between FA and 1-year follow-up ASIA grade in this group of patients. On the other hand, in patients with TSCI no significant correlation was found at time of injury or 1-year follow-up. Finally, dividing the high cervical cord into 3 segments revealed that FA obtained from the lower segment showed the strongest correlation with admission ASIA scores. Our results indicate that FA measured at the high cervical cord, rostral to the injury site, might be a potential biomarker of neural injury following acute CSCI for diagnosing injury severity and prognosticating long-term functional outcomes.

We decided to measure FA at the high cervical cord (C1-2), a location remote from the site of injury, due to the better visualization of cord boundary at that level. The injury site is often difficult to delineate due to susceptibility artifacts, and/or it might not provide an accurate estimate of neural injury due to edema, hemorrhage, or bone fragments contaminating the diffusion signal. Our rationale is supported by prior studies in preclinical animal models showing a correlation between changes to diffusion anisotropy remote from the injury site and injury severity as well as locomotor and electrophysiological function. . $^{17-10,12,16-18,20}$
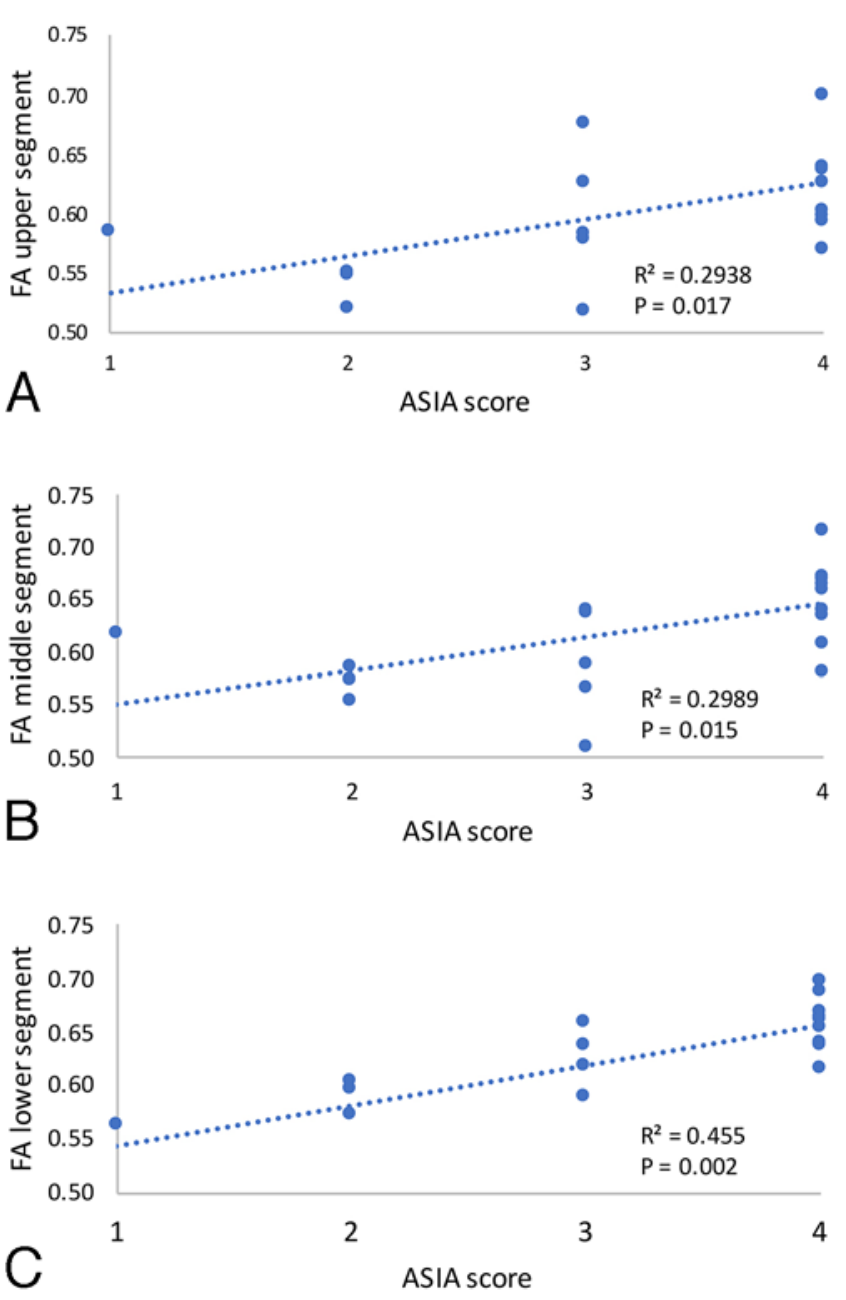

FIG. 6. Graphs showing significant correlation between FA measured at each segment (upper, middle, and lower) of the high cervical cord (C1-2) and ASIA grade at the time of admission for the group of patients with only $\mathrm{CSCI}(\mathrm{N}=19)$. The ASIA grades were converted into numerical values, with ASIA A corresponding to the numerical value of 1 . The strength of the correlation progressively increased in the rostrocaudal direction $(p=0.017, p=0.015$, and $p=0.002$ for upper, middle, and lower segments of high cervical cord [C1-2], respectively).

Similar findings have been reported in human subjects with varying severities of injury and time since injury, suggesting that retrograde neural degeneration occurs at locations remote to the injury site. ${ }^{4,6,11,15,27,34,35}$ Together, these results point to extensive alterations throughout the spinal cord in the aftermath of SCI that are not detected on conventional MRI. Furthermore, the measurement of high cervical anisotropy can identify preserved white matter tracts, leading to a possible role in monitoring recovery following therapeutic interventions.

The presence of a correlation between FA measured at the high cervical cord (C1-2) and admission ASIA shows the superiority of DTI in predicting injury severity compared with conventional MRI. Our results are similar to previous studies showing cord abnormalities on diffusionweighted imaging (DWI) in the presence of unremarkable findings on conventional MRI in humans and animal models. ${ }^{5,18,32,38}$ The finding of a significant relationship between 
FA and injury severity indicates the presence of changes in the spinal cord remote to the site of injury, which supports the rationale for evaluating DTI parameters at distant locations. ${ }^{32}$

We observed a significant correlation between degree of neurological impairment as measured by admission ASIA and FA at the high cervical cord, which indicates severity of cord injury reflected in the measurement of DTI parameters. This is in line with previous findings showing regions remote from the injury site being impacted during the acute phase of the SCI. ${ }^{7,14,26,29,30}$ It appears that the initial traumatic insult to the spinal cord leads to pathophysiological changes along the length of the cord that might have long-term implications. Based on the understanding that FA measures the extent of restricted diffusion, the correlation seen between ASIA and FA values could be indicative of increasing neuronal injury in the high cervical section of the cord. Our findings are similar to results published previously showing lower FA values with increasing neurological impairment. ${ }^{34}$

The present analysis included patients with cervical and/or thoracic injuries. We decided to include TSCI based on the reasoning that changes induced in the upper cervical cord might not be limited to the injuries to the cervical cord. Our results showed a nonsignificant relationship between degree of neurological impairment as measured by ASIA and FA for the overall sample. However, correlation analysis performed following exclusion of patients with TSCI showed significant correlation for the CSCI patients. Similarly, we observed a significant correlation between high cervical FA and ASIA at 1-year follow-up for the cohort containing only cervical injuries, whereas the addition of patients with thoracic injuries changed the correlation to a nonsignificant one. Together, these results indicate that the distance between the upper cervical cord and the location of injury could influence the pathophysiological changes occurring remotely in the upper cervical cord. It would be interesting for future studies to perform FA measurements in different segments of the spinal cord in relation to the site of injury and to have them normalized to the distance between site of injury and the site of measured FA. This might be limited by susceptibility artifacts if the measured FA site is too close to the site of injury, but it can define the most suitable site for measuring FA for different SCI levels.

The calculation of FA for the high cervical cord involved averaging the FA obtained from multiple axial slices spanning the length of $\mathrm{C} 1$ and $\mathrm{C} 2$. On closer inspection, a general trend of increasing FA in the rostrocaudal direction was observed. Our findings are similar to results reported previously that found the highest value for FA around C2/3 and could be due to better alignment of white matter fibers at $\mathrm{C} 2$ compared to $\mathrm{C} 1 .{ }^{37}$ The finding of significant differences between DTI parameters obtained from the $\mathrm{C} 1$ versus $\mathrm{C} 2$ level indicates that within the upper cervical cord $\mathrm{C} 2$ might be a better location for drawing ROIs. The use of $\mathrm{C} 2$ is also supported by prior analysis showing a decrease in FA in the cranial as well as caudal direction, due to the presence of increased gray matter at the level of the cervicomedullary junction in the rostral direction and at the cervical enlargement in the caudal direction. ${ }^{37}$

\section{Study Limitations}

Our findings should be understood in the context of relevant limitations associated with this study. The present study was retrospective in design with a small number of patients, and a large proportion of them had ASIA grade D on admission, which could have influenced our results. There are difficulties associated with performing statistical analysis because of the low number of patients in the setting of additional heterogeneity due to the severity and location of the injury. Further compounding the situation is the lack of published studies establishing the numbers needed for performing statistical analysis of DTI parameters in the SCI population. Nonetheless, we believe that our results provide pilot data on the expected effect, which should guide future research efforts involving larger data sets. To add to the previous point, we think that inclusion of more patients with ASIA A and B would probably serve to strengthen the correlation between FA and ASIA grades observed in this study.

We were unable to perform 1-year follow-up ASIA assessment in a number of patients, which might have contributed to the weaker relationship observed between high cervical FA and ASIA grade at 1-year follow-up. The present study only imaged the patients with SCI during the acute phase, which does not allow for longitudinal monitoring of microstructural changes within the spinal cord associated with recovery from SCI. Unfortunately, due to small sample size, loss to follow-up, higher proportions of ASIA D at time of injury, and no improvement in the ASIA grade at 1-year follow-up for almost all patients, it is difficult to determine whether FA is superior in predicting ASIA score at 1-year follow-up compared to the presenting ASIA grade at the time of injury.

\section{Conclusions}

The diffusion anisotropy measured at the high cervical region (C1-2) correlates significantly with injury severity and long-term follow-up only in patients with CSCI. Estimation of FA from the lower portion of the high cervical cord (C1-2) shows a stronger correlation with ASIA at time of injury. In patients with TSCI, no significant correlation is seen between FA and injury severity on longterm follow-up. The distance between the site of injury in patients with TSCI and the measurement site for FA (C1-2) might be a contributing factor to our findings. In patients with cervical cord injury, overall, the relationship between FA and injury severity as well as long-term outcome highlights the possibility that high cervical cord FA can be a potential imaging biomarker for neural injury and for monitoring recovery in patients following acute CSCI. The findings of the present study, in addition, have led to the start of a prospective study at our institution aimed at achieving higher follow-up rates and longer follow-up duration for patients with SCI in order to overcome some of the limitations noted in this paper.

\section{Acknowledgments}

This work was partly supported by the corresponding author's VA Rehabilitation R\&D Grant and an endowment from the Bryon Riesch Paralysis Foundation. 


\section{References}

1. Agosta F, Laganà M, Valsasina $P$, Sala $S$, Dall'Occhio L, Sormani MP, et al: Evidence for cervical cord tissue disorganisation with aging by diffusion tensor MRI. Neuroimage 36:728-735, 2007

2. Basser PJ, Mattiello J, LeBihan D: MR diffusion tensor spectroscopy and imaging. Biophys J 66:259-267, 1994

3. Bondurant FJ, Cotler HB, Kulkarni MV, McArdle CB, Harris JH Jr: Acute spinal cord injury. A study using physical examination and magnetic resonance imaging. Spine (Phila Pa 1976) 15:161-168, 1990

4. Cheran S, Shanmuganathan K, Zhuo J, Mirvis SE, Aarabi $\mathrm{B}$, Alexander MT, et al: Correlation of MR diffusion tensor imaging parameters with ASIA motor scores in hemorrhagic and nonhemorrhagic acute spinal cord injury. J Neurotrauma 28:1881-1892, 2011

5. Cohen-Adad J, Benali H, Hoge RD, Rossignol S: In vivo DTI of the healthy and injured cat spinal cord at high spatial and angular resolution. Neuroimage 40:685-697, 2008

6. Cohen-Adad J, El Mendili MM, Lehéricy S, Pradat PF, Blancho S, Rossignol S, et al: Demyelination and degeneration in the injured human spinal cord detected with diffusion and magnetization transfer MRI. Neuroimage 55:1024-1033, 2011

7. Deo AA, Grill RJ, Hasan KM, Narayana PA: In vivo serial diffusion tensor imaging of experimental spinal cord injury. J Neurosci Res 83:801-810, 2006

8. Ellingson BM, Kurpad SN, Li SJ, Schmit BD: In vivo diffusion tensor imaging of the rat spinal cord at 9.4T. J Magn Reson Imaging 27:634-642, 2008

9. Ellingson BM, Kurpad SN, Schmit BD: Ex vivo diffusion tensor imaging and quantitative tractography of the rat spinal cord during long-term recovery from moderate spinal contusion. J Magn Reson Imaging 28:1068-1079, 2008

10. Ellingson BM, Kurpad SN, Schmit BD: Functional correlates of diffusion tensor imaging in spinal cord injury. Biomed Sci Instrum 44:28-33, 2008

11. Ellingson BM, Ulmer JL, Kurpad SN, Schmit BD: Diffusion tensor MR imaging in chronic spinal cord injury. AJNR Am J Neuroradiol 29:1976-1982, 2008

12. Ellingson BM, Ulmer JL, Prost RW, Schmit BD: Morphology and morphometry in chronic spinal cord injury assessed using diffusion tensor imaging and fuzzy logic. Conf Proc IEEE Eng Med Biol Soc 1:1885-1888, 2006

13. Flanders AE, Spettell CM, Tartaglino LM, Friedman DP, Herbison GJ: Forecasting motor recovery after cervical spinal cord injury: value of MR imaging. Radiology 201:649655,1996

14. Ford JC, Hackney DB, Alsop DC, Jara H, Joseph PM, Hand $\mathrm{CM}$, et al: MRI characterization of diffusion coefficients in a rat spinal cord injury model. Magn Reson Med 31:488-494, 1994

15. Freund P, Schneider T, Nagy Z, Hutton C, Weiskopf N, Friston $\mathrm{K}$, et al: Degeneration of the injured cervical cord is associated with remote changes in corticospinal tract integrity and upper limb impairment. PLoS One 7:e51729, 2012

16. Jirjis MB, Kurpad SN, Schmit BD: Ex vivo diffusion tensor imaging of spinal cord injury in rats of varying degrees of severity. J Neurotrauma 30:1577-1586, 2013

17. Jirjis MB, Valdez C, Vedantam A, Schmit BD, Kurpad SN: Diffusion tensor imaging as a biomarker for assessing neuronal stem cell treatments affecting areas distal to the site of spinal cord injury. J Neurosurg Spine 26:243-251, 2017

18. Kim JH, Loy DN, Liang HF, Trinkaus K, Schmidt RE, Song SK: Noninvasive diffusion tensor imaging of evolving white matter pathology in a mouse model of acute spinal cord injury. Magn Reson Med 58:253-260, 2007

19. Kim JH, Loy DN, Wang Q, Budde MD, Schmidt RE, Trinkaus K, et al: Diffusion tensor imaging at 3 hours after traumatic spinal cord injury predicts long-term locomotor recovery. J Neurotrauma 27:587-598, 2010

20. Kim JH, Song SK, Burke DA, Magnuson DS: Comprehensive locomotor outcomes correlate to hyperacute diffusion tensor measures after spinal cord injury in the adult rat. Exp Neurol 235:188-196, 2012

21. Kulkarni MV, McArdle CB, Kopanicky D, Miner M, Cotler $\mathrm{HB}$, Lee KF, et al: Acute spinal cord injury: MR imaging at 1.5 T. Radiology 164:837-843, 1987

22. Le E, Aarabi B, Hersh DS, Shanmuganathan K, Diaz C, Massetti J, et al: Predictors of intramedullary lesion expansion rate on MR images of patients with subaxial spinal cord injury. J Neurosurg Spine 22:611-621, 2015

23. Li XH, Li JB, He XJ, Wang F, Huang SL, Bai ZL: Timing of diffusion tensor imaging in the acute spinal cord injury of rats. Sci Rep 5:12639, 2015

24. Loy DN, Kim JH, Xie M, Schmidt RE, Trinkaus K, Song SK: Diffusion tensor imaging predicts hyperacute spinal cord injury severity. J Neurotrauma 24:979-990, 2007

25. Miyanji F, Furlan JC, Aarabi B, Arnold PM, Fehlings MG: Acute cervical traumatic spinal cord injury: MR imaging findings correlated with neurologic outcome-prospective study with 100 consecutive patients. Radiology 243:820 827,2007

26. Nevo U, Hauben E, Yoles E, Agranov E, Akselrod S, Schwartz M, et al: Diffusion anisotropy MRI for quantitative assessment of recovery in injured rat spinal cord. Magn Reson Med 45:1-9, 2001

27. Petersen JA, Wilm BJ, von Meyenburg J, Schubert M, Seifert B, Najafi Y, et al: Chronic cervical spinal cord injury: DTI correlates with clinical and electrophysiological measures. J Neurotrauma 29:1556-1566, 2012

28. Rajasekaran S, Kanna RM, Karunanithi R, Shetty AP: Diffusion tensor tractography demonstration of partially injured spinal cord tracts in a patient with posttraumatic Brown Sequard syndrome. J Magn Reson Imaging 32:978-981, 2010

29. Schwartz ED, Chin CL, Shumsky JS, Jawad AF, Brown BK, Wehrli S, et al: Apparent diffusion coefficients in spinal cord transplants and surrounding white matter correlate with degree of axonal dieback after injury in rats. AJNR Am J Neuroradiol 26:7-18, 2005

30. Shanmuganathan K, Gullapalli RP, Zhuo J, Mirvis SE: Diffusion tensor MR imaging in cervical spine trauma. AJNR Am J Neuroradiol 29:655-659, 2008

31. Shanmuganathan K, Zhuo J, Chen HH, Aarabi B, Adams J, Miller C, et al: Diffusion tensor imaging parameter obtained during acute blunt cervical spinal cord injury in predicting long-term outcome. J Neurotrauma 34:2964-2971, 2017

32. Shen H, Tang Y, Huang L, Yang R, Wu Y, Wang P, et al: Applications of diffusion-weighted MRI in thoracic spinal cord injury without radiographic abnormality. Int Orthop 31:375-383, 2007

33. Tu TW, Kim JH, Yin FQ, Jakeman LB, Song SK: The impact of myelination on axon sparing and locomotor function recovery in spinal cord injury assessed using diffusion tensor imaging. NMR Biomed 26:1484-1495, 2013

34. Vedantam A, Eckardt G, Wang MC, Schmit BD, Kurpad SN: Clinical correlates of high cervical fractional anisotropy in acute cervical spinal cord injury. World Neurosurg 83:824828,2015

35. Vedantam A, Eckardt G, Wang MC, Schmit BD, Kurpad SN: High cervical fractional anisotropy as an imaging marker for spinal cord injury. Neurosurgery 61 (Suppl 1):167-170, 2014

36. Vedantam A, Jirjis MB, Schmit BD, Budde MD, Ulmer JL, Wang MC, et al: Diffusion tensor imaging and tractography in Brown-Sequard syndrome. Spinal Cord 50:928-930, 2012

37. Wheeler-Kingshott CA, Hickman SJ, Parker GJ, Ciccarelli O, Symms MR, Miller DH, et al: Investigating cervical spinal 
cord structure using axial diffusion tensor imaging. Neuroimage 16:93-102, 2002

38. Zhang J, Jones M, DeBoy CA, Reich DS, Farrell JA, Hoffman PN, et al: Diffusion tensor magnetic resonance imaging of Wallerian degeneration in rat spinal cord after dorsal root axotomy. J Neurosci 29:3160-3171, 2009

\section{Disclosures}

The authors report no conflict of interest concerning the materials or methods used in this study or the findings specified in this paper.

\section{Author Contributions}

Conception and design: Kurpad, Shabani, Kaushal. Acquisition of data: Shabani, Kaushal. Analysis and interpretation of data: Shabani, Kaushal. Drafting the article: Shabani, Kaushal. Critically revising the article: all authors. Reviewed submitted version of manuscript: all authors. Approved the final version of the manuscript on behalf of all authors: Kurpad.

\section{Supplemental Information \\ Videos}

Video Abstract. https://vimeo.com/315450140.

\section{Correspondence}

Shekar N. Kurpad: Medical College of Wisconsin, Milwaukee, WI.skurpad@mcw.edu. 